\section{Regards sur l'économie allemande}

Bulletin économique du CIRAC

$102 \mid 2011$

Varia

\title{
BTP allemand : restructuration et nouveaux enjeux
}

Solène Hazouard

\section{OpenEdition}

Journals

Édition électronique

URL : http://journals.openedition.org/rea/4333

DOI : 10.4000/rea.4333

ISBN : 978-2-8218-1134-8

ISSN : 1965-0787

Éditeur

CIRAC

Édition imprimée

Date de publication : 31 octobre 2011

Pagination : 29-34

ISSN : 1156-8992

Référence électronique

Solène Hazouard, "BTP allemand : restructuration et nouveaux enjeux », Regards sur l'économie allemande [En ligne], 102 I octobre 2011, mis en ligne le 01 octobre 2013, consulté le 03 mai 2019 URL : http://journals.openedition.org/rea/4333 ; DOI : 10.4000/rea.4333 


\section{BTP allemand : restructuration et nouveaux enjeux}

\section{Solène Hazouard}

La deuxième journée du BTP allemand (Bauwirtschaftstag), intitulée "Transformation de l'Allemagne - Opportunités et enjeux pour le secteur du BTP », s'est tenue le 29 septembre dernier à Berlin. Elle était organisée par l'association fédérale du secteur (Bundesvereinigung Bauwirtschaft) qui, par l'intermédiaire de ses fédérations membres, réunit près de 300000 entreprises artisanales du BTP au sens large du terme (services de nettoyage de bâtiments et secteur métallurgique inclus). A cette journée d'échanges avec les entreprises et fédérations du secteur étaient conviés des responsables politiques : le ministre de l'environnement Norbert Röttgen, le président du parti social-démocrate, Sigmar Gabriel, ainsi que le secrétaire général du parti libéral, Christian Lindner. Parmi les thèmes abordés, l'efficience énergétique, qui place le secteur du BTP au cœur des enjeux sociétaux du moment. Un défi à relever notamment pour les trois grands de la branche que sont Hochtief, Bilfinger et Strabag Köln : en misant sur l'internationalisation et, plus encore, la diversification, ces majors du bâtiment ont résisté à la vague d'insolvabilité qui avait frappé la branche au début des années 2000. Ce sont désormais des entreprises de services (financiers, de maintenance...) qui, pour plus de sécurité, ont délégué leur cœur de métier aux nombreuses TPE du secteur.

\section{Un secteur en voie de normalisation après le boom de l'Unité}

Le secteur du BTP constitue un vaste ensemble délivrant divers types de prestations. Selon la définition de l'Office fédéral des statistiques, il se scinde en deux grands sous-ensembles : l'activité principale du BTP (Bauhauptgewerbe) et l'aménagement (Ausbaugewerbe). L'activité principale du BTP comprend la construction de bâtiments, les travaux d'infrastructures, les travaux de démolition, les travaux préparatoires de chantiers et autres activités spécialisées du bâtiment (charpente et toiture, échafaudages). L'aménagement regroupe pour sa part les installations techniques (électricité, eau, gaz, chauffage, aération, climatisation) et les travaux d'aménagement divers (plâtres, peintures, sols, portes et fenêtres). Mais il comprend aussi l'activité des promoteurs immobiliers et la viabilisation de terrains.

Le chiffre d'affaires de l'activité principale du BTP avait reculé entre 1995 (116,8 milliards $€$ ) et 2005 , pour se stabiliser par la suite aux alentours de 80 milliards $€$. Les 81,9 milliards $€$ réalisés en 2010 se répartissaient ainsi : construction de logements (32\%), contrats publics (32\%), construction de bâtiments pour les entreprises $(22 \%)$ et travaux d'infrastructures pour les entreprises $(14 \%)$.

La même tendance est observable pour le secteur dans son ensemble : selon la fédération patronale de l'industrie allemande du BTP (Hauptverband der Deutschen Bauindustrie, HDB), la part de la création de valeur ajoutée rapportée à l'économie allemande est passée de $7,1 \%$ en 1994 à $3,7 \%$ en 2010 , les investissements rapportés au PIB, de $14,3 \%$ à 9,5\%. L'Unité allemande et l'effort de reconstruction des infrastructures est-allemandes comme la réhabi-

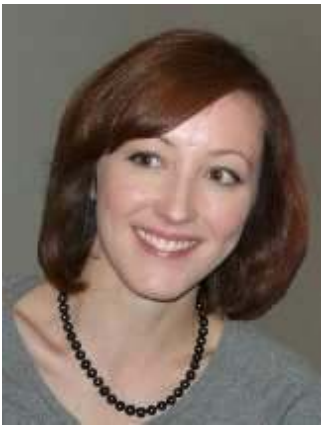

Solène Hazouard, Ingénieur d'études au CIRAC

Deux grands sous-ensembles

Stabilisation du secteur depuis 2005 
BTP allemand : $1^{\mathrm{er}}$ investisseur dans l'UE

1995-2005 : une crise structurelle du marché

Un secteur atomisé, dominé par les PME... litation de l'habitat avaient hypertrophié ce secteur dont le niveau d'activité est depuis revenu à une certaine normalité.

A l'échelle européenne en revanche, l'Allemagne présentait le plus haut niveau d'investissements dans le secteur en 2010 (249,8 milliards $€$ ), suivie de près par la France $(247,7$ milliards $€)$. Si la part du BTP dans la population active occupée est passée de $15 \%$ en 1995 à $8 \%$ en 2010 dans les Länder de l'est dans le fil de sa normalisation, celle-ci est restée stable aux alentours de $6 \%$ à l'ouest. Par ailleurs, 6,4 \% des insolvabilités déclarées en Allemagne reviennent actuellement au secteur du BTP. Ce taux est faible, comparé aux pics enregistrés en 1985 et sur la période 1995-2001 (quelque 15 à 16\%).

La crise du secteur, déclenchée pendant la seconde moitié des années 1990, résulte parallèlement d'une baisse des marges. En effet, les grands groupes du BTP avaient commencé voici quelque temps à déléguer les travaux de chantier à des entreprises externes moins chères : bien souvent des TPE ne versant pas de salaires conventionnels (c'est-à-dire non affiliées à un syndicat patronal de branche) ou des entreprises étrangères. Mais en procédant ainsi, les grandes entreprises du bâtiment se sont extraites de leur cœur de métier, ce qui a abouti à une restructuration du marché et à une vague d'insolvabilité touchant notamment de plein fouet de grands noms du secteur comme Philipp Holzmann à Francfort en 2002 ou Walter Bau à Augsbourg en 2005 (respectivement numéros 2 et 4 du secteur quelques années plus tôt).

Dans un premier temps, cette crise structurelle a été masquée par le boom qui avait immédiatement suivi la réunification. Touchant l'est comme l'ouest, il avait été entretenu par la combinaison de plusieurs facteurs : la vétusté du parc immobilier à l'est, l'augmentation de la population ouest-allemande de plus de 6 millions de personnes (originaires d'Europe de l'Est et des nouveaux Länder), ainsi qu'un régime fiscal particulièrement incitatif à l'investissement immobilier (voir REA 64/03). Les entreprises de l'ouest investissaient à l'est, générant des surcapacités qui ont fait chuter d'abord les prix, puis le marché pour finalement provoquer une vague d'insolvabilité. En l'espace de dix ans, le nombre de salariés du secteur a ainsi été pratiquement divisé par deux : il s'élève désormais à 700000 personnes.

L'activité principale du BTP est un marché atomisé que se partagent près de 74000 entreprises de dix salariés en moyenne. Depuis le pic de plus de 80000 entreprises, atteint en 2000 à la fin de la période de croissance conjoncturelle, le nombre d'entreprises du secteur recule d'environ $1 \%$ par an. Les TPE de moins de 20 salariés ont gagné du terrain en l'espace de 15 ans : elles constituent désormais $90,2 \%$ des entreprises du secteur (contre $77,5 \%$ en 1995), emploient $46,4 \%$ des salariés de la branche et réalisent $34,2 \%$ du chiffre d'affaires (contre respectivement 27,3 et $23,4 \%$ en 1995 ).

Du début de 2009 à la fin de l'année 2010, ce sont elles qui ont bénéficié en priorité du programme conjoncturel fédéral (Konjunkturpaket), dont les 21 milliards $€$ dédiés au BTP et à ses secteurs connexes ont aidé à financer de petits projets de construction (rénovation d'écoles, de jardins d'enfants, et autres établissements communaux). Avec un ralentissement toutefois depuis le printemps 2010 et la fin du critère d'additionnalité (Zusätzlichkeitskriterium) : l'attribution des aides n'étant plus conditionnée par la mise sur pied de nouveaux projets, communes et Länder ont dès lors utilisé les fonds du Konjunturpaket pour financer essentiellement des projets existants.

Sur le total des entreprises du secteur, près d'un tiers sont de très petites structures réalisant un CA compris entre 17500 et $100000 €$ (soit 1,4\% du CA de la branche). Seulement $0,2 \%$ des entreprises réalisent un CA supérieur à 50 millions $€$, contribuant à $28 \%$ au CA total. Les trois principaux groupes allemands de BTP sont Hochtief (20,5 milliards $€$ de CA en 2009), Bilfinger Berger $(10,4$ milliards $€)$ et Strabag Köln $(4,1$ milliards $€)$. Hochtief, détenu à plus de $50 \%$ par le groupe espagnol ACS, occupe le huitième rang mondial et le troi- 
sième rang européen, derrière les deux leaders français que sont Vinci et Bouygues.

Les partenaires sociaux du secteur sont, du côté patronal, la fédération HDB pour les grandes entreprises et les entreprises du Mittelstand, et la fédération centrale du BTP allemand (Zentralverband Deutsches Baugewerbe, ZDB) pour les entreprises artisanales, avec en face le syndicat d'industrie IG BAU (BTPagriculture-environnement). En toute indépendance de l'Etat et selon le modèle de partenariat social allemand, ces trois organismes négocient et établissent conjointement les conventions collectives de branche.

Comme toujours en Allemagne, les intérêts sectoriels sont également représentés par nombre de fédérations professionnelles, dont la Bundesvereinigung Bauwirtschaft et l'association des entreprises moyennes du BTP (Bundesvereinigung Mittelständischer Bauunternehmen, BVMB), qui réunit des entreprises familiales du Mittelstand. A cela s'ajoutent des fédérations régionales et spécialisées selon les métiers. Le BTP comprend en effet toute une série de métiers : différents périmètres sont ainsi pris en considération dans l'élaboration des statistiques relatives au secteur, ce qui complique la vision d'ensemble.

Au niveau politique, le ministère fédéral en charge des Transports, du BTP et des Infrastructures (BMVBS) a élaboré pour la $17^{\mathrm{e}}$ législature, en place depuis 2009, l'initiative «Strategiedialog Bauwirtschaft » visant à renforcer les échanges entre l'association allemande de matériaux de construction (Bundesverband Baustoffe - Steine und Erden, BBS), la BVMB, la HDB, IG Bau, et la ZDB. L'opinion publique voyant bien souvent dans le secteur du BTP des conditions d'emploi précaires et de maigres perspectives d'évolution, ce dialogue stratégique avec les acteurs de la profession a pour objectif d'améliorer l'image de la branche et de la faire ainsi concorder avec ses performances réelles en termes de création de valeur. Par ce biais, le gouvernement poursuit la démarche entreprise lors de la législature précédente, et qui avait abouti à la création d'une charte pour le secteur (Leitbild Bau), élaborée en concertation avec 11 fédérations couvrant l'ensemble de la chaîne de création de valeur.

\section{Défis économiques, environnementaux et sociétaux}

Au sein de l'activité principale du BTP, la construction de logements (neufs et rénovation) a vu ses investissements croître à nouveau en 2010 , retrouvant son niveau de 2006. Plusieurs facteurs expliquent cette évolution positive : la stabilité du marché de l'emploi, la poursuite des mouvements migratoires dans les agglomérations, le faible niveau des taux d'intérêts appliqués en Allemagne aux crédits à la construction de logements, le changement de stratégie de placement des investisseurs et la croissance modérée des coûts de construction.

Dans ce contexte encourageant, l'aide fiscale aux mesures de rénovation d'immeubles d'habitation a été toutefois refusée par le Bundesrat en juillet dernier, l'assemblée des Länder craignant par là même une nouvelle détérioration des finances publiques. Le président de la Bundesvereinigung Bauwirtschaft précisait à ce sujet que "les investissements dans le BTP ainsi stimulés sont créateurs d'emplois pour les PME artisanales du secteur sises dans la région et renforcent considérablement la conjoncture intérieure, engendrant ainsi des recettes fiscales et des cotisations sociales supplémentaires ॥.

En matière de construction de logements neufs, le marché a enregistré une baisse de $40 \%$ entre 2006 et 2009 . Avec la construction de 1,7 logement neuf pour 1000 habitants, le pays faisait alors office de lanterne rouge en Europe. Cela s'explique notamment par la suppression de la prime d'accès à la propriété (Eigenheimzulage) en 2006, qui devait être notamment compensée deux ans plus tard par une aide au remboursement de l'emprunt immobilier s'inscrivant dans le système d'allègement fiscal de la retraite complémentaire ('Wohn-Riester'). Lancée en 1996 et destinée à favoriser l'accession à la pro-
... mais coordonné par un réseau dense de fédérations

Depuis 2009, un « dialogue stratégique » de branche

Récente reprise de la construction de logements 
Le BTP des entreprises tiré par les projets d'infrastructure

Des contrats publics grevés par le déséquilibre des finances publiques

Stratégie des grands groupes : internationalisation et orientation accrue vers les services annexes

Un secteur particulièrement touché par la pénurie de main-d'œuvre qualifiée priété des catégories sociales moins aisées, l'Eigenheimzulage servait surtout à subventionner l'activité de l'épargne logement. La plupart des bénéficiaires ne réalisant aucun projet immobilier, elle avait surtout un effet redistributif. Au début des années 2000, certains économistes lui attribuaient même une part de responsabilité dans la piètre performance du marché du logement allemand (voir REA 64/03). La reprise actuelle de la construction de logements reste ainsi à relativiser : elle correspond davantage à une situation de rattrapage.

La construction de bâtiments pour les entreprises reste, elle, en recul depuis le début de la crise (-9\% de CA en 2010), du fait notamment de la baisse du taux d'utilisation des capacités de production dans l'industrie induite par la récession. En revanche, les travaux d'infrastructures (+4\% en 2010) sont tirés par les projets du secteur ferroviaire, de l'industrie pétrolière et de l'énergie. Ils bénéficient également des programmes de soutien conjoncturel adoptés en 2009.

Troisième et dernier volet de l'activité principale du BTP : les investissements publics, dont le volume est déterminé en premier lieu par l'état des finances publiques. Or, l'Etat fédéral, les Länder et les communes doivent réduire désormais leurs dépenses pour rééquilibrer leur budget, et ce alors que le sous-financement durable des infrastructures (chemins de fer, routes, voies navigables) devient critique pour le territoire économique allemand dans son ensemble. II est vrai que depuis les années 1990, l'essentiel de l'effort avait été consenti au développement des infrastructures dans les nouveaux Länder, ce dont à pâti la modernisation de celles de l'ouest. Particulièrement sensible est la situation budgétaire des communes qui supportent à elles seules $60 \%$ des dépenses publiques du secteur du BTP ; près des trois quarts de la construction de bâtiments et près de la moitié des travaux d'infrastructures leur reviennent.

Les deux grands du secteur, Hochtief et Bilfinger, ont historiquement établi leur croissance sur le cœur de métier de la construction, essentiellement sur le marché allemand. Celui-ci dégageant des marges de plus en plus réduites, les deux sociétés ont développé ces dernières années une offre de services dans l'industrie, l'énergie et la gestion d'immeubles qui, partie de zéro en 2002, constitue désormais $90 \%$ du résultat d'exploitation de Bilfinger par exemple. Une stratégie complétée par un processus d'internationalisation efficace, entamé dès les années 1930 par le groupe Hochtief.

Cela étant, face à la faiblesse des investissements publics dans le BTP en Allemagne, le directeur de la fédération patronale HDB, Michael Knipper, rappelle qu'un marché intérieur fort constitue une base nécessaire au bon fonctionnement des entreprises allemandes. Dès lors, il exhorte les responsables politiques à investir dans le secteur par le biais de grands projets. La polémique autour du projet d'extension de la gare de Stuttgart (Stuttgart 21) n'explique qu'en partie la frilosité des acteurs politiques, le principal frein résidant dans le manque de moyens financiers du côté des pouvoirs publics.

Mais la principale entrave au bon fonctionnement du secteur réside dans la pénurie de main-d'œuvre, aussi bien à l'est qu'à l'ouest. Depuis 2004, le besoin annuel de 4500 jeunes ingénieurs du BTP n'est plus couvert, le nombre de diplômés stagnant à 3 000. La demande de main-d'œuvre est forte aussi pour ce qui est des ouvriers qualifiés : fin 2010, $25 \%$ des postes proposés restaient vacants dans le secteur. La situation est encore plus tendue parmi les maîtresmaçons, où seulement $60 \%$ des départs en retraite sont couverts par l'embauche de jeunes suffisamment formés.

De plus, malgré les évolutions techniques, le travail dans le secteur reste très éprouvant physiquement. Le report de l'âge légal de départ à la retraite à 67 ans devient dès lors un enjeu de plus à gérer pour les entreprises. Car aujourd'hui, l'âge effectif moyen de départ à la retraite des salariés du BTP est de 60 ans.

La branche dispose en outre de salaires minima légaux, introduits dès 1997 dans le cadre de la Loi sur les travailleurs détachés (Arbeitnehmerentsende- 
gesetz, AEntG) de 1996 qui visait à éviter le « dumping social » lié à l'ouverture des marchés européens (voir REA 71/2005). Depuis la revalorisation intervenue le $1^{\text {er }}$ juillet 2011 , ces salaires minima légaux se situent entre $9,75 €$ et $13 €$ de I'heure. Ils feront à nouveau l'objet d'une réévaluation globale au $1^{\mathrm{er}}$ janvier 2012, puis partielle au $1^{\mathrm{er}}$ janvier 2013.

Salaires minima horaires dans le secteur du BTP

\begin{tabular}{|l|cc|cc|cc|}
\hline & \multicolumn{2}{|c|}{ Ouest } & \multicolumn{2}{c|}{ Est } & \multicolumn{2}{c|}{ Berlin } \\
& Groupe 1 & Groupe 2 & Groupe 1 & Groupe 2 & Groupe 1 & Groupe 2 \\
$1^{\text {er }}$ septembre 2010 & $10,90 €$ & $12,95 €$ & $9,50 €$ & - & $10,90 €$ & $12,75 €$ \\
$1^{\text {er } j u i l l e t ~ 2011 ~}$ & $11,00 €$ & $13,00 €$ & $9,75 €$ & - & $11,00 €$ & $12,85 €$ \\
$1^{\text {er }}$ janvier 2012 & $11,05 €$ & $13,40 €$ & $10,00 €$ & - & $11,05 €$ & $13,25 €$ \\
$1^{\text {er }}$ janvier 2013 & $11,05 €$ & $13,70 €$ & $10,25 €$ & - & $11,05 €$ & $13,55 €$ \\
\hline
\end{tabular}

Source : Zentralverband Deutsches Baugewerbe. Le salaire minimum du groupe 1 vaut pour l'accomplissement de travaux simples de construction et de montage, ainsi que pour les activités de maintenance et d'entretien de machines et appareils pour lesquels aucune qualification particulière n'est requise. Le salaire minimum du groupe 2 s'applique aux travaux techniquement limités (accomplissement partiel des missions d'un poste ou activités spécialisées). Depuis le $1^{\text {er }}$ septembre 2009, le salaire du groupe 2 ne constitue plus le salaire minimum légal au sens de l'article 2 , $\S 1$ de la Loi AEntG que dans les Länder de l'ouest et à Berlin. A l'est, le salaire du groupe 2 résulte de la convention collective non obligatoire sur les salaires et les rémunérations des apprentis dans le secteur du BTP qui s'applique aux nouveaux Länder, à l'exception de Berlin. Ce salaire se monte à 10,25 $€$ de l'heure.

Avec le « concept énergie » (Energiekonzept), le gouvernement fédéral a défini en septembre 2010 neuf critères pour un approvisionnement en énergie alliant respect de l'environnement, fiabilité et maîtrise des coûts. La rénovation énergétique des bâtiments existants et la construction de bâtiments à haut rendement énergétique constituent l'un des points nodaux du programme. Avec des évolutions jusqu'à présent encourageantes: l'Office fédéral des statistiques révèle que, malgré une augmentation de $15 \%$ de la surface habitable depuis 1995 , le besoin énergétique des ménages a reculé de près de $10 \%$, et les émissions de $\mathrm{CO}_{2}$ de près de $20 \%$. Toutefois, pour atteindre l'objectif d'une maison passive, des progrès doivent encore être réalisés dans le domaine des matériaux isolants.

En matière législative, le règlement portant sur l'économie d'énergie (Energieeinsparverordnung, EnEV), entré en vigueur en 2002, a été révisé en 2009 dans l'objectif de réduire de $30 \%$ les besoins énergétiques en chauffage et eau chaude. Pour les constructions nouvelles s'applique en outre la loi sur le chauffage par les énergies renouvelables (Erneuerbare-Energien-Wärmegesetz, EEWärmeG), qui fait obligation légale aux propriétaires de bâtiments de recourir en partie à ces nouvelles énergies pour le chauffage. Mais il est avant tout nécessaire d'agir dans les immeubles anciens, pas ou peu concernés actuellement par l'EnEV : 80 \% des bâtiments en Allemagne ont en effet été construits avant 1978. Par l'intermédiaire de la banque publique KfW, le gouvernement fédéral met ainsi à disposition des propriétaires de logements des subventions et crédits à faibles taux d'intérêt en vue d'améliorer l'efficience énergétique des bâtiments. Un second programme offre aux maîtres d'ouvrage des crédits à taux avantageux pour la construction de logements neufs à faible dépense énergétique.

Par le biais de subventions et de crédits, la banque publique KfW soutient également l'adaptation du logement des personnes âgées. Cet enjeu est particulièrement important dans le contexte du vieillissement de la population allemande. En effet, ces deux prochaines décennies verront le nombre de personnes de plus de 80 ans augmenter de 110000 chaque année. Dès lors, la possibilité accordée aux personnes âgées de vivre plus longtemps en autonomie dans leur logement devrait grandement contribuer à alléger aussi la charge pesant sur l'assurance dépendance.

ASSOCIANT LES DÉFIS DE LA BRANCHE aux enjeux environnementaux et sociétaux actuels, la fédération ZDB émet trois préconisations dans le cadre de sa campagne "Impulsions pour la construction de logements » : développer les incita-
Enjeux environnementaux...

... et sociétaux 
tions fiscales via une élévation du taux d'amortissement de 2 à $4 \%$ pour la construction de logements locatifs, améliorer les conditions cadre pour la modernisation énergétique et les bâtiments neufs de remplacement, et promouvoir l'adaptation du logement des personnes âgées, notamment via la poursuite du programme d'aide de la banque publique KfW. Si ces préconisations visent à soutenir la construction de logements dans un secteur par ailleurs confronté à l'atonie des commandes publiques et à une pénurie de main-d'œuvre qualifiée, le contexte démographique et les nouvelles normes environnementales devraient néanmoins grandement contribuer à stimuler l'activité du BTP dans les décennies à venir. Pour leur part, les investissements des entreprises dans le BTP connaissent de fortes variations d'un secteur à l'autre. Le potentiel de croissance future semble ainsi reposer sur les services, et notamment le commerce, l'hôtellerie, les transports et les services financiers qui ces dernières années ont investi quatre fois plus dans le BTP que le secteur secondaire.

\section{Indications bibliographiques}

- Bundesarchitektenkammer, Bundesingenieurkammer, Bundesverband Baustoffe - Steine UND ERDEN, BUNDESVEREINIGUNG MITTELSTÄNDISCHER BAUUNTERNEHMEN, GEFMA DEUTSCHER VERBAND FÜR FACILITY MANAGEMENT, HAUPTVERBAND DER DEUTSCHEN BAUINDUSTRIE, INDUSTRIEGEWERKSCHAFt BAUEN-AgRAR-UMWELt, Verband BERATENDER INGENIEURE, VERBAND DEUTSCHER

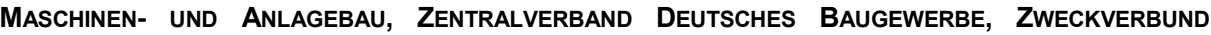
OStDEutscher BAUVERBÄNDE (eds.), Leitbild Bau. Zur Zukunft des Planens und Bauens in Deutschland - eine gemeinsame Initiative der deutschen Bauwirtschaft, mars 2009

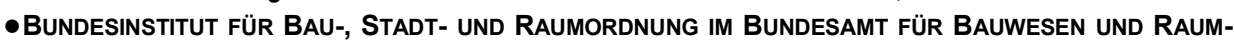
ORDNUNG, Bericht zur Lage und Perspektive der Bauwirtschaft 2010, Bundesinstitut für Bau-, Stadt- und Raumordnung im Bundesamt für Bauwesen und Raumordnung, Bonn, novembre 2010

- Bundesverband Baustoffe - Steine und ERden, Bundesvereinigung Mittelständischer BAUUNTERNEHMEN, HAUPTVERBAND DER DEUTSCHEN BAUINDUSTRIE, INDUSTRIEGEWERKSCHAFT Bauen-AgraR-Umwelt, Zentralverband Deutsches Baugewerbe, Strategiedialog Bau Forderungen der Bauwirtschaft, Parlamentarierbrief, septembre 2011

- Bundesvereinigung BAUWIRTschaft, Stärken bündeln, Zukunft bauen, Kompetenz beweisen Die Bundesvereinigung Bauwirtschaft im Geschäftsjahr 2010, Bundesvereinigung Bauwirtschaft, Berlin, mars 2011

- Gabel M., «La finance allemande dans l'impasse immobilière? », Regards sur l'économie allemande, $\mathrm{n}^{\circ} 64$, décembre 2003

- KFW BANKENGRUPPE, «Altersgerechter Umbau des Wohnungsbestandes und KfW-Förderung », Akzente, $\mathrm{n}^{\circ} 51$, septembre 2011

-Streck S., WischHof K., Materialband zum Leitbild Bau, Wuppertal/Hambourg, janvier 2009

-Zander O., Hauptverband der Deutschen Bauindustrie (eds), Tarifsammlung für die Bauwirtschaft 2011/2012 - Gewerbliche Arbeitnehmer, Poliere und Angestellte. Lohn- und Gehaltstarife, Rahmentarife, Sozialkassentarife, Verfahrenstarife, Tabellenwerk, Elsner, Dieburg, 2011

-Zentralverband Deutsches Baugewerbe, Baumarkt 2010, Berlin, mai 2011

-Zentralverband Deutsches BaugewERBE, Geschäftsbericht 2010, Berlin, février 2011

- www.bauindustrie.de , www.bauwirtschaftstag.de ; www.bilfinger.com; www.bmu.de ; www.bmvbs.de ; www.bv-bauwirtschaft.de ; www.bvmb.de ; www.hochtief.de ; www.kfw.de ; www.zdb.de. 